Revue européenne des sciences sociales

European Journal of Social Sciences

XLI-125 | 2003

Index de la Revue européenne des sciences sociales

(1995-2002)

\title{
Index des matières
}

\section{(2) OpenEdition}

\section{Journals}

Édition électronique

URL : http://journals.openedition.org/ress/557

DOI : $10.4000 /$ ress. 557

ISSN : 1663-4446

Éditeur

Librairie Droz

\section{Édition imprimée}

Date de publication : 1 janvier 2003

Pagination : $39-44$

ISBN : 2-600-00844-6

ISSN : 0048-8046

Référence électronique

" Index des matières », Revue européenne des sciences sociales [En ligne], XLI-125 | 2003, mis en ligne le

30 novembre 2009, consulté le 04 mai 2019. URL : http://journals.openedition.org/ress/557 ; DOI :

$10.4000 /$ ress. 557 


\section{INDEX DES MATIÈRES}

actant 1557

acteur 1556, 1557, 1558, 1559, 1560,

$1561,1562,1564,1565$

action politique 1525

action sociale 1570

agent 1557, 1558, 1561, 1562, 1563

Allemagne 1239

alphabétisation 1463

analogie 1509

analogies 1508, 1516, 1518

anatomique, originalité 1476

angoisse 1540

animal 1480,1481

animalité $1467,1468,1477$

anthropologie 1269, 1476

anthropologie politique 1393

anthropologie des sciences et des techniques 1594

apprentissage, organisation 1327

argumentation 1292, 1333, 1335, 1336,1340

argumentativiste, point de vue 1453

Aristote 1473

artificialisme 1245

audience 1460

autobiographie 1566

autorité, argumentation 1340

avenir 1580

balancier sociologique 1597

banal 1480

Benjamin, Walter 1238

Bergson H. 1477

bibliographie analytique et chronologique 1253

bibliographiques notes 1278

biologie 1527

bolchevisme 1305

Bortkiewicz, L. von 1520

Boudon, Raymond 1401, 1402, 1409 , $1410,1413,1414,1417$
Bourdieu, Pierre 1367

Bramois 1329

Braudel, Fernand 1223

Bunge, Mario 1439

capitalisme 1490, 1578

cardinal 1517

carnet de bord 1368, 1522

causalité, analyse empirique 1404

changement 1370, 1373, 1374, 1376, 1379, 1380, 1381, 1383, 1384.

changement social 1379, 1381, 1418

Chevalier, Michel 1378

civilisation thermo-industrielle 1352

clinique psychiatrique de Genève 1366

codes 1356,1583

cœur 1252

comédien 1559

commerce 1362

communauté 1586

communication 1328, 1388, 1397, $1448,1459,1462,1464$

compétition 1365

complément, traitement 1554

complexité du social, pensée 1227

complexité en sociologie 1591

comportement 1474, 1563

conceptuelles, révisions 1540

congrès international de philosophie de Genève (1904) 1317

connaissance, 1267, 1272, 1394, 1509, 1585

connaissance, objectivité 1422

construction des savoirs 1392

construction sociale 1593

contradiction, nouvelle approche 1334

contradictions 1576

coopération 1365

correspondance 1447

correspondance Walras-Pareto 1501

croyances 1524 
culture $1466,1566,1570$

culture de classe, théorie des 1259

décision 1319

délinquance féminine 1250

démocratie 1233, 1234, 1398

démographie 1373

départements académiques 1242

désenchantement du monde 1237, 1238,1574

désillusion 1257

développement technique 1348, 1350

déviance 1250

De Viti 1494

digressions 1226, 1249, 1323, 1360

discours 1454, 1572

dispositifs de mémoire objectivée 1393

don 1309,1310

don, théorie anthropologique 1308

Dreyfus, affaire 1314

droit 1285, 1291, 1295, 1332, 1440, $1530,1542,1551,1559$

Dupuit, Jules 1363

Durkheim 1306, 1307, 1550

dynamique 1384

école 1411

écologie 1243

économétrie 1341, 1534

économie 1225, 1282, 1377, 1443, 1486, 1489, 1502, 1505, 1579

économie normative 1293

économie politique 1363,1488

économique, principe 1494

économique, théorie 1441

économiques, données 1442

écriture sumérienne 1386

éducation 1409

égalité 1247,1378

électeurs 1456

encyclopédie 1521

enseignements 1452

entrepreneur parétien 1491

environnement 1358, 1526

épistémologie piagétienne 1322

épistémologie sociale 1587

équilibre 1499
Etat 1381, 1514, 1569

éthique 1222, 1481, 1525, 1527, 1528, 1529

éthique des sciences 1531

étude des sciences 1596

Europe de l'Est 1535

évolution 1472

évolutionnisme 1377

existence 1244

Explication en sociologie L' 1321

famille 1411

femme 1250

féodalisme, transition 1315

fiction 1577

Foucault 1229

fourmi 1563

Fournier 1301

France 1239

futur 1375

Gardin, Jean-Claude 1546

génétique 1473

génétique, code 1482

génie génétique 1356

géographie 1374, 1375, 1376, 1451, $1518,1561,1562$

géographie humaine 1336

globalisation 1365

Granger 1229

Halévy 1317

héritage 1256

hétérogénéité ontologique du social 1591

heuristiques, postures 1269

hiérarchies 1247

histoire 1231, 1265, 1377, 1521, 1600

histoire de la sociologie 1568

historien 1368, 1522

holisme 1310, 1425

homme 1431, 1432, 1435, 1480, 1508

homo complexus 1478

homo geographicus 1436

homo oeconomicus 1441, 1541

homo sapiens 1541

homo sociologicus 1437

humain, capital 1444 
humanité 1467, 1468, 1477, 1479

iconorrhée 1387

identité 1482

identité nationale 1522

identité personnelle 1598

immortalité 1583

impensable, peur 1538, 1543

impostures 1484

impôt 1363

incertitude 1319

inconnu 1540

indicateurs culturels 1457

individu 1585

individus et structures 1597

individualisme 1310, 1416

inégalité sociale 1410, 1411, 1413

inégalités 1247,1415

information 1358, 1391, 1394, 1397, 1398

informations 1395

intentionnalité 1586

interdisciplinarité 1229

intérêt public 1290

interfaces 1391

interfaces, effets d' 1354

intériorité 1471,1475

interventions 1445

Judaïsme 1302

juridicisation de l'ordre politique 1530

juridique 1528

juridique, argumentation 1331

juridique, discours 1513

justice 1487

Kafka, Franz 1519

langage 1403

langue 1438

lectures 1226, 1249, 1323, 1360, 1445

légitimité politique 1232

liberté 1221, 1240, 1530

libre concurrence 1488

livres 1369

logique 1286

logique du social 1598

loi $1281,1285,1286,1295,1296$ loi des géographes 1290

lois 1294,1417

malentendus 1484

mammifères 1470

marché, modèle et théorie 1412

marchés, structure 1601

Marx 1324, 1364

mathématique 1406, 1495

mathématiques, traces 1372

Maunier, René 1312

Mauss, Marcel 1298, 1299, 1300, 1301, 1302, 1304, 1305, 1306, $1307,1308,1309,1310,1311$, 1312,1313

mécanique industrielle 1363

mécanismes sociaux 1497

médias 1392, 1455, 1457, 1458, 1466

mémoire 1389, 1396

mémoires 1395

mercantilisme libéral 1362

métaphore 1510, 1513, 1519

métaphores 1508, 1512, 1514, 1515, 1517,1518

méthodologiques, normes 1280

Mill 1344

Miserabilis, Docteur 1263

modèle 1417

modernité 1237,1326

modernité politique 1316

monde, labyrinthe 1283

mondialisation 1365

moral, statut 1481

morale 1526

musulmane, fillette 1331

natation 1331, 1333

nature 1224

nature du politique 1599

naturel 1271

néant 1275

négatif, plénitude 1554

négationnisme 1314

négociations territoriales 1284

Nietzsche 1438

nihilisme 1356

Nivat, Georges 1320

nomologique 1275 
norme $1284,1286,1292,1586$

norme constitutionnelle 1297

norme, degré zéro 1255

observation 1322

objectivité, science 1589

oiseaux 1470

opinion publique 1382

optimalité 1506

organes 1564

organisme 1475

oubli 1261

Pantaleoni 1494

Pareto, Bibliothèque de 1230

Pareto, Vilfredo 1224, 1255, 1317 , $1344,1438,1491,1492,1493$, 1494, 1495, 1496, 1497, 1498, $1499,1500,1501,1502,1503$, $1504,1505,1506,1507,1523$

Passeron, Jean-Claude 1252, 1253, 1258,1277

pensée 1371

pensée artificielle 1390

pensée sociologique 1321

persuasion de masse 1452

peuple 1261

philosophie 1473,1600

Piaget, Jean 1321, 1359

plage 1563

pluridisciplinarité 1269

poésie 1509

poïétique 1510

polémiques 1445

politique $1231,1380,1438,1526$, $1528,1529,1532$

politique, expertise 1533

politiques, arguments 1337

politiques publiques 1381

Populo, Mister 1263

Portmann, Adolf 1468, 1469, 1472

positivisme 1595

poste suisse 1327

post-modernité 1449

pratiques 1572

précipice 1539

prédication «nostrologique 1599 préférences 1465

pression sociale 1382

présupposés 1399

preuves 1549

prix relatifs 1601

probabilités, calcul 1319

processus sociaux 1498

professionnalité sociologique 1260

programme sociologique 1596

progrès 1347

psychologique, parallélisme 1359

puissance 1530

raison 1296

raison, débat 1276

raisons 1420,1423

raisonnement, 1266, 1274

Rappard, W. E. 1324

rationalisation 1237

rationalisme 1425

rationalisme critique 1419,1424

rationalité $1254,1433,1441$

réactions 1226, 1249, 1323, 1360

réalisme scientifique 1588

réalité, principe 1273

recherche scientifique 1350

règles 1289,1558

relation 1564

relativisme 1326

religion 1581

remarques interlocutoires 1400

rencontres internationales de Genève 1320

réseaux socio-techniques 1351

responsabilité 1221

rêve 1511

revenu 1444

révolution 1235

révolution scientifique 1590

rhétorique anthropologiquement fondée 1224

richesse 1444, 1493

Rogoff-Ramsoy, Nathalie 1414

Romano 1521

Sacré 1532

savant, le $1257,1260,1262$ 
savoir 1395, 1446, 1546, 1580

savoirs 1294, 1529

science 1246,1287

science des mœurs 1421

sciences du langage 1462

science économique, mutation 1358

science juridique 1543

sciences 1343, 1345, 1349, 1431, $1432,1455,1508$

sciences historiques 1549

sciences humaines 1330, 1383

sciences sociales 1280, 1325, 1342, $1404,1452,1453,1553,1556$, 1561

scientifique, pensée 1510

secret, espace du 1267

sélection 1247

sentiments 1423

sentiments moraux 1421

serre, effet de 1352

Serres 1229

sida 1461

silence 1459

silhouettes, mise en scène 1446

Simmel, Georg 1545

singularités 1294

Smith, Adam 1315

social 1287

social, changement 1379, 1381

sociale, enquête 1450

sociale, recherche 1403, 1506

sociales, anomies 1365

sociales, normes 1280

socialisation scolaire 1248

sociaux, faits 1547

société 1244, 1508, 1576

société de l'information 1385, 1537 , $1572,1573,1574,1575,1576$, $1578,1579,1580,1581,1582$, 1594

sociétés occidentales contemporaines 1415

sociologie 1225, 1228, 1259, 1265, $1269,1270,1273,1379,1406$, 1407, 1437, 1496, 1497, 1518, $1564,1566,1567,1573$

sociologie, diversité 1339

sociologie classique 1550 sociologie de la connaissance 1344, 1399

sociologie de la réception 1264, 1268 sociologie de la culture 1258

sociologie de l'expérience esthétique 1258

sociologie des sciences 1552, 1592, 1595,1600

sociologie française, histoire 1278

sociologie mathématique

sociologie religieuse 1303, 1316

sociologique, connaissance 1512

sociologique, enquête 1533

sociologique, réflexion 1295

sociologique, regard 1291

sociologisme 1595

sociologue 1251, 1548, 1567, 1584

sociologue, itinéraire 1277

sociologues 1544

socio-technique, limites 1385

somme 1358

Spencer 1524

spoliation 1364

Starobinski, Jean 1320

statique 1384

stratège 1559

structure, notion 1408, 1566

structure énonciative 1454

subjectivité, science 1589

surveillance 1582

symbolisme 1306

système social 1496

technique 1244,1577

technique, fonction mythogène 1390

technique, sphère 1354

technique autonome 1353

techniques 1343

techniques, quasi-objets 1357

technologie contemporaine 1357

technologies de la communication 1583

technologies de l'information 1391

technologies du vivant 1583

technosciences 1346

territorialisation 1349

théorie de la communication 1449

théorie de la description 1591 
théorie de l'équilibre social 1500

théorie de l'évolution 1473

théorie des formes socio-historiques 1318

théorie des jeux 1409

théorie juridique 1560

théorie neo-ricardienne 1520

théorie sociale 1448

Theuth, mythe 1387

Tocqueville 1324

Toscane 1492

troubles 1434

Tweety 1433

types 1570

université 1536, 1544, 1565

utopie $1487,1537,1574$

valeur 1254

valeurs 1422 vérité 1438

vérités, Etat 1338

vide, horreur du 1224

vide symbolique 1539

vie 1471,1483

ville 1233,1562

Völkerpsychologie 1304

vulgarisation 1355

Walras 1486, 1487, 1488, 1489, 1490, $1501,1502,1503,1504,1505$

Weber, Max 1231, 1232, 1234, 1235 , $1238,1239,1240,1254,1316$, 1550

Wechselwirkung 1545

Wittgenstein 1479

X-crise 1534

zoologie 1469 